\title{
POST TENSIONED FLOORS AS AN ECONOMIC ALTERNATIVE I] EGYPTIAN MARKET (CASE STUDY)
}

Gouda Mohamed, Tharwat Sakr, Ghada Mohamed

\begin{abstract}
Post tensioned "PT "floors are one of the most widely used systems for building construction all over the world. Such systems have too many behavior, construction and economic benefits over other systems used for relativitely long span floor system. In Egypt, the use of PT systems still has market and economir constraints related to practice and leading to very limited use of such superior system. This paper aims at investigating in-depth the different merits of PT systems considering behavior, design, construction and economic issues. In addition a numerical study for comparison of the costs with the RC flat slab constructir which is the most commonly used system in the Egyptian market. Economic span of PT systems and the saving in concrete, rebar costs as balanced to the additional PT strands, ducts and accessoriness's are investigated. Results indicated the advantages of using PT floor systems especially for relatively large spans and multistory construction where time plays an important role. Spans more than 8.3 meters, PT sy proved to be more economic according to the Egyptitian market practice. This economic span becomes slightly less in case of more live loads.
\end{abstract}

